\title{
A New Approach to the Design of Helical Shape Memory Alloy Spring Actuators
}

\author{
Esuff Khan and Sivakumar M. Srinivasan \\ Department of Applied Mechanics, IIT Madras, Chennai 600036, India \\ Correspondence should be addressed to Sivakumar M. Srinivasan, mssiva@iitm.ac.in
}

Received 21 July 2011; Revised 24 October 2011; Accepted 25 October 2011

Academic Editor: Xianglong Meng

Copyright ( $) 2011$ E. Khan and S. M. Srinivasan. This is an open access article distributed under the Creative Commons Attribution License, which permits unrestricted use, distribution, and reproduction in any medium, provided the original work is properly cited.

\begin{abstract}
Shape memory alloys (SMAs) are smart materials that have the ability to recover their original shape by eliminating residual deformations, when subjected to adequate temperature rise (Shape memory effect). This special behavior attracts the use of SMAs as efficient stroke/force actuators. Most of the engineering applications require helical springs as actuators and proper design of SMA helical spring actuators is very important. In the traditional design approach of SMA spring (Waram, 1993), only strain between linear zones was considered in order to simplify the design and to improve the fatigue life. Only modulus difference between high-temperature and low-temperature phase was utilized, and the transformation strain was not considered as the total transformation strain will be more and will degrade the performance of actuator. In the present design, we have shown that transformation strain can be restricted by using hard stops and the partial transformation strain can be used to improving the capacity of SMA spring actuator. A comparison of the traditional design approach of SMA spring and the proposed design procedure has been made to give an idea of its effect on the design and the related parameters.
\end{abstract}

\section{Introduction}

Shape memory alloys (SMAs) are smart materials which undergo solid-to-solid phase transformations under thermomechanical loading exhibiting special intrinsic properties such as the pseudoelasticity and the shape memory effect (SME). The shape memory effect of SMAs provides possibilities of using it as actuators [1]. Unlike other known actuators, the SMA actuators are nonlinear in behavior because of the nature of SMA as a material. This paper presents a simple procedure for the design of helical SMA spring actuators, taking into account their nonlinear behavior and the introduction of hard stops.

SMAs generally exist in two phases: austenite, a hightemperature phase, and martensite, a low-temperature phase. Martensite is soft in nature, can undergo finite deformations during loading, and leaves residual strains when unloaded. During loading, the stress and strain response is essentially linear until it reaches a critical stress value. At this critical stress value, it could enter into transformation zone where twinned martensite gets converted to detwinned martensite, undergoing large strains for small stress increments. This strain is called the transformation strain. At any point, if the temperature is increased well beyond the austenite temperature, the SMA recovers the residual strain (phase transformation from martensite to austenite), producing an actuation stroke. During this phase transformation process of an SMA, large loads and/or displacements can be generated in a relatively short period of time making this component an interesting mechanical actuator.

Because of such remarkable properties, SMAs have found a number of applications in different areas [2]. Han et al. [3] showed how SMA spring actuator can be used to enhance the buckling capacity of columns. Lee and Lee [4] explored the application of SMA spring actuator in active catheter medical device. Spinella and Dragoni [5] showed that the actuator performance can be improved by using the hollow SMA springs. They proved that by emptying the inefficient material from the wire center, the hollow section features a lower mass, lower cooling time, and lower heating energy than its solid counterpart for given strength, stiffness, and deflection. Thus, it becomes necessary to design the SMA 


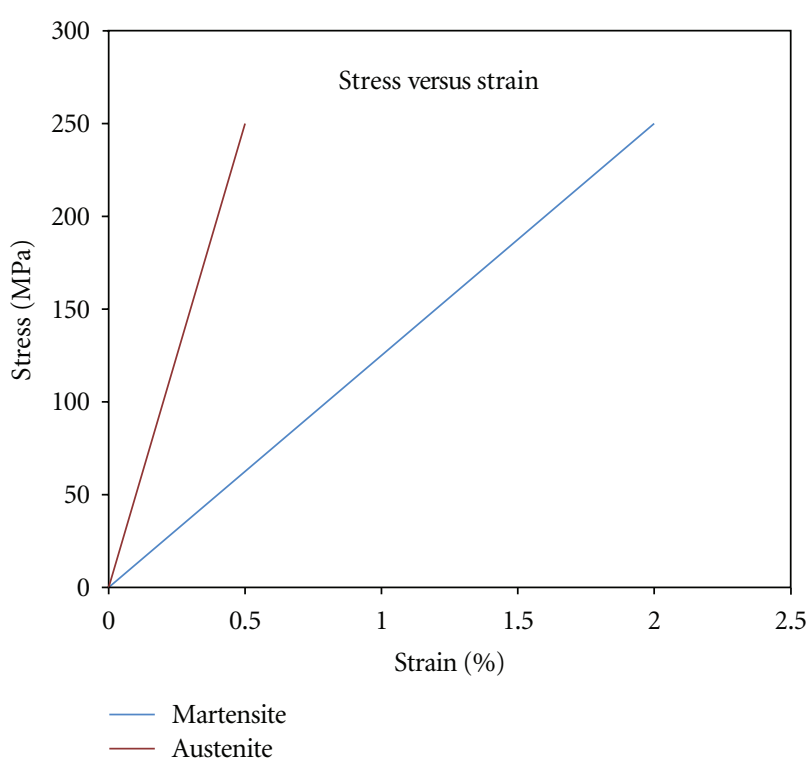

FIGURE 1: Stress versus strain response in linear zone.

actuator appropriate to an application to allow for efficient performance.

In literature, the design approaches proposed so far have assumed a linear mechanical response for both martensite and austenite [2-6]. Essentially transformation induced change in modulus is assumed in these approaches to be responsible for the stroke in the actuator. Waram's design approach [6] for SMA spring is one such popular design approach that considers mainly the modulus difference between the martensite and austenite phases in the design as shown in Figure 1. The reason for such a design approach being popular could be that in the transformation region, SMA undergoes large strains for small increment of stress value. In addition, allowing high deformations that occur in this transformation regime may render degradation in its functional as well as structural performance. In Waram's design, the martensite phase strain is restricted in design to allow for a very good fatigue life. The stroke obtainable from such designs is low compared to that which can be achieved by allowing for transformation strains.

Also, it should be stressed that unlike in most other actuators, the material of SMA actuators is nonlinear in mechanical response beyond a critical stress. The response beyond this stress is involved in the effective actuator action and that could make the design complex because of the nonlinearity involved. In this paper, we propose an approach which can utilize the partial transformation strain into the spring design. Transformation strain is considered to be restricted by using the external hard stop, so that only partial detwinning takes place during loading. By including the partial transformation strain into the design, the actuation capacity of SMA helical spring may improve without compromising significantly on its fatigue life.

In this paper, the design parameters are analyzed with the consideration of transformation strains. The nonlinear behavior of martensite phase is idealized as an elastoplastic response to reduce the complexity in the formulation and is compared with the traditional linear approach. Linear approach is first presented in the next section (Section 2). Then, the nonlinear analysis and design approach is discussed in Section 3. The comparison between the two approaches based on different design parameters is made in Section 4 before making concluding remarks on the need for nonlinear approach to the design of helical SMA springs.

\section{Linear Approach (Waram's Design [6])}

In the standard design procedure, the aim is to arrive at the wire diameter, $d$, the spring diameter, $D$, and the number of turns, $n$, for a spring that will deliver the required force, $P$, and a stroke, $S$, in a full actuation cycle. The appropriate values of shear moduli, $G_{h}$, in the hot and, $G_{l}$, in the cold states, the maximum allowable shear stress, $\tau_{c}$, in the austenitic state, and the limit on shear strain, $\gamma$, in the cold state are the input parameters in this design procedure. The procedure is described below [6], in brief.

The maximum shear stress allowable in the austenite state, $\tau_{c}$, provided from fatigue considerations puts a constraint on maximum allowable force on the spring, $P_{\max }$. The shear stress in the wire and the force on the spring can be related using

$$
\tau_{c}=\frac{W 8 C P_{\max }}{\pi d^{2}},
$$

where the Spring Index, $C=d / D$, generally taken to be between 6 and 10 and $W$, is the Wahl correction factor.

From (1), it is possible to find out the required wire diameter of the spring given the maximum allowable design load. Equation (2) is an expression that allows one to decide on the number of coils using " $d$," " $D$," $S$ and the allowable shear strain difference $\Delta \gamma=\left(\gamma_{l}-\gamma_{h}\right)$ where $\gamma_{l}$ is the maximum low-temperature martensite shear strain allowable and $\gamma_{h}$ is the high temperature shear strain. The limit on the low temperature martensitic shear strain is to ensure adequate cycle life without functional degradation.

The number of coils $(n)$ can be calculated by

$$
n=\frac{S d}{\pi \Delta \gamma D^{2}} .
$$

However, a closer look at the design equations reveals that once the value of $P$ and $S$ are chosen, the required number of coils $(n)$ gets automatically fixed without requiring an externally imposed limit on the shear strain recommended in the standard design.

The deflection of the spring $(\delta)$ in hot or cold state, assuming the material to be elastic is given by

$$
\delta=\frac{8 P D^{3} n}{G d^{4}}
$$

where $P$ is the force exerted by the spring and $G$, the rigidity or shear modulus in the appropriate state.

The stiffness $(K)$ can be calculated as

$$
K=\frac{G d^{4}}{8 D^{3} n},
$$




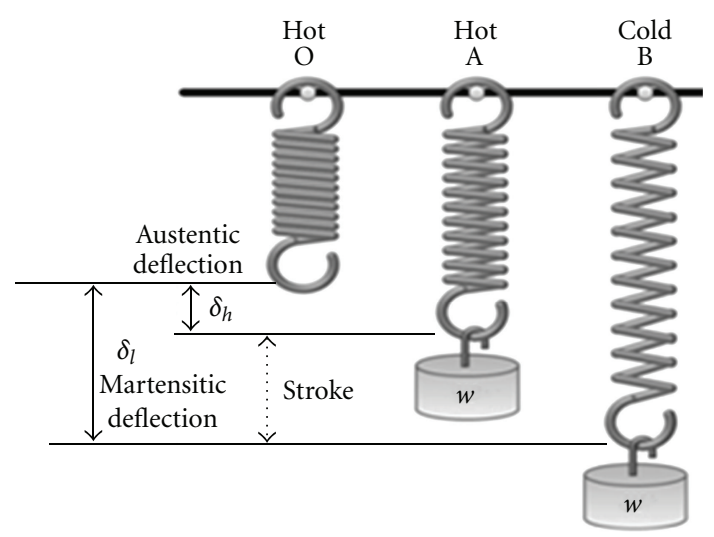

FIGURE 2: Schematic diagram spring actuator.

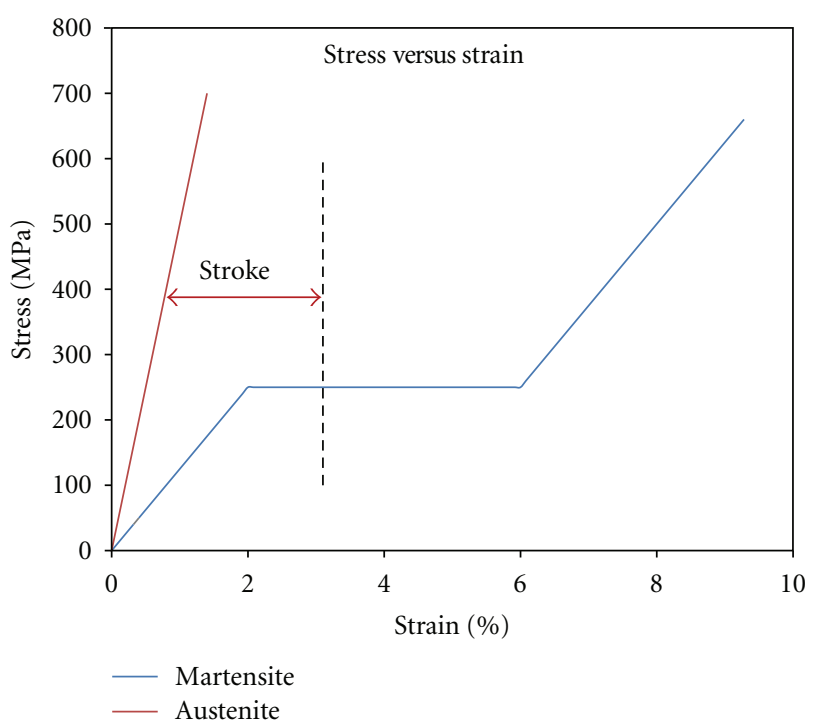

FIgURE 3: Idealized stress versus strain response of SMA.

where $G$ pertains to the appropriate shear modulus cold, $G_{l}$ or hot, $G_{h}$.

Therefore, the total load to stroke relationship can be obtained for the spring in the cold and the hot state as shown in Figure 2. $K_{l}$ and $K_{h}$ stand for low- and high-temperature stiffness.

The maximum possible stroke $\left(S_{\max }\right)$ can now be expressed as

$$
S_{\max }=P\left[\frac{1}{K_{l}}-\frac{1}{K_{h}}\right]=\frac{8 P D^{3} n}{d^{4}}\left[\frac{1}{G_{l}}-\frac{1}{G_{h}}\right] .
$$

In the above approach, the stroke depends upon the difference in the reciprocal of the shear moduli of the martensite and the austenite phases.

\section{Proposed Nonlinear Approach}

In this approach, partial transformation strain is utilized. Considering the total transformation strain will degrade the cyclic life of actuator. The transformation strain can be

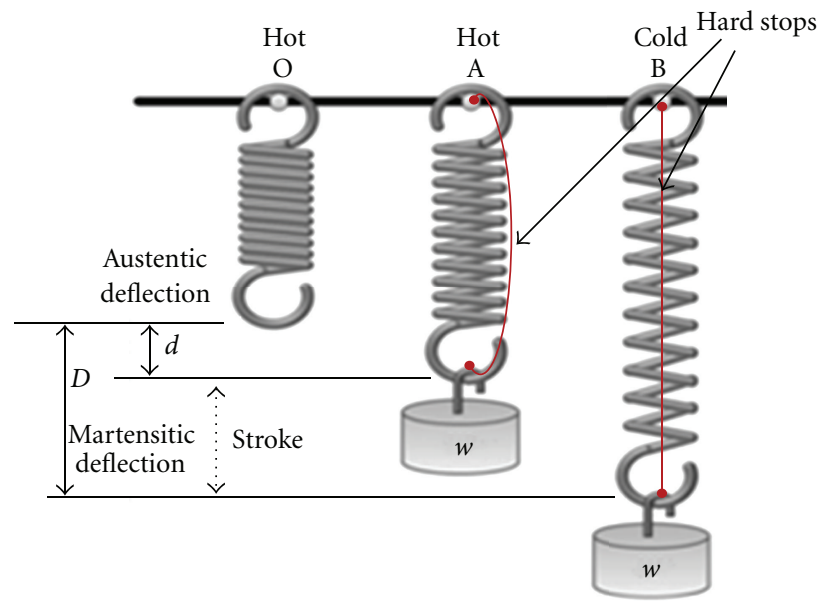

FIGURE 4: SMA spring actuator with hard stop.

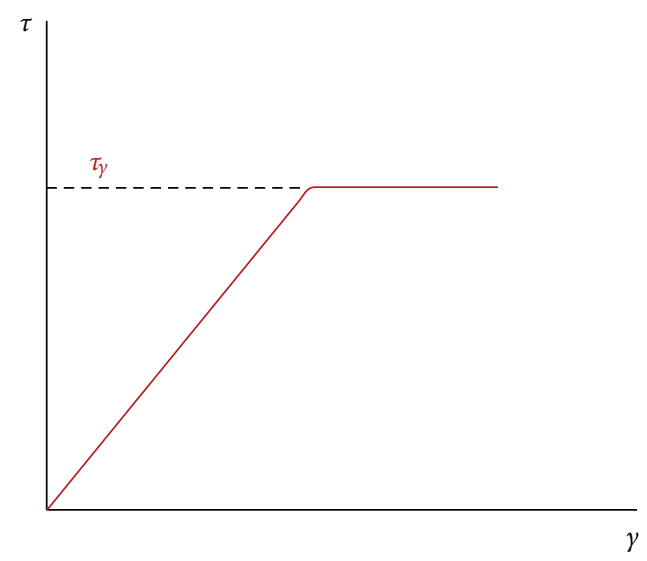

FIgURE 5: Simplified martensite phase response.

restricted using hard stops, which are highly stiff materials. By using these hard stops as shown in Figures 3 and 4, now in the martensite phase, the material undergoes large strain, and appropriate actuation will give more stroke compared to linear approach. Figure 5 is the simplified martensite phase behavior of SMA which will consist of both twinned and detwinned martensite. In Figure 6 the stress distribution over the wire cross-section is shown. Till $\tau$ reaches $\tau_{y}$, the material behaves linearly, and then transformation starts from the outer region and move toward the inner core.

At the Maximum Elastic Torque:

$$
\begin{gathered}
T_{y}={ }^{J} \tau_{y}=\frac{1}{2} \pi c^{3} \tau_{y}, \\
\phi_{y}=\frac{L \gamma_{y}}{c},
\end{gathered}
$$

where $T_{y}, \tau_{y}, \gamma_{y}$ are the maximum torque, stress, and strain, respectively, at which transformation starts, $J$, the polar of moment of inertia, and $\phi_{y}$ the angle of twist at maximum stress. 


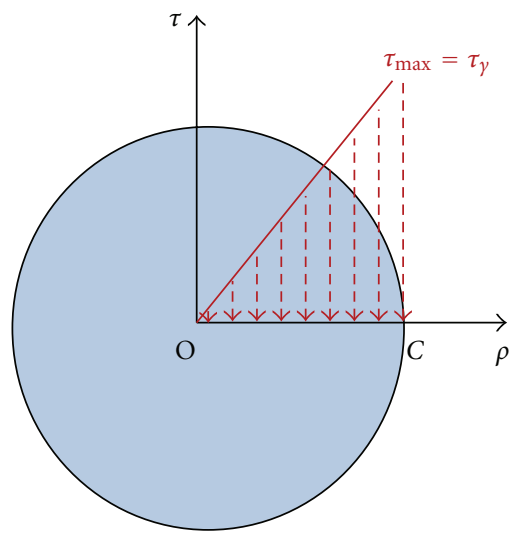

(a) Linear

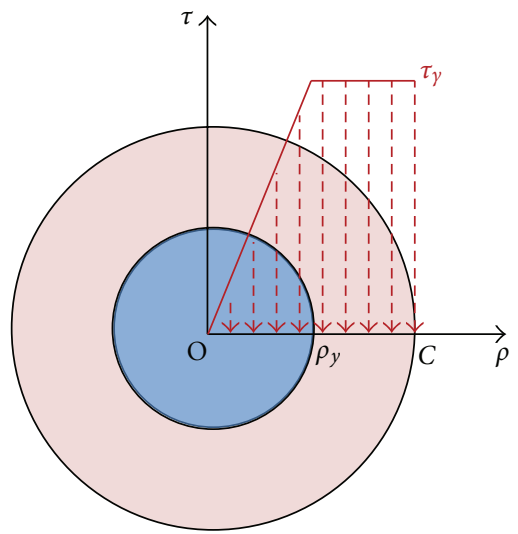

(b) Non-Linear

FIGURE 6: Stress distribution over the wire cross section.

Practically, a helical spring is subjected to both torsional shear stress and direct shear stress but a closer look at the stress values gives an understanding that the torsional shear stress is much higher $(2 \times$ spring index $)$ compared to the direct shear stress, and therefore, the direct shear stress effect is neglected in this study.

As the torque is increased, a transformed region develops around an elastic core:

$$
\begin{gathered}
\frac{\rho_{y}}{\gamma_{y}}=\frac{\rho}{\gamma} \\
T=\frac{2}{3} \pi c^{3} \tau_{y}\left(1-\frac{1}{4} \frac{\rho_{y}^{3}}{c^{3}}\right)=\frac{4}{3} T y\left(1-\frac{1}{4} \frac{\phi_{y}^{3}}{\phi^{3}}\right) .
\end{gathered}
$$

Also the above equation can be simplified as:

$$
P=\frac{2}{3} \frac{\pi}{C} c^{2} \tau_{y}\left(1-\frac{1}{4} \frac{\gamma_{y}^{3}}{\gamma^{3}}\right) .
$$

From the above equation, it is possible to calculate the wire diameter $(d=2 * c)$ once the total strain value is provided. The value of the spring index $(C)$ is assumed to be 6.As $\rho_{y} \rightarrow 0$, the torque approaches a limiting value, (see Figure 7):

$$
T_{p}=\frac{4}{3} T_{y}=\text { critical torque. }
$$

Since there is no warping in circular prismatic members, (2) can also be used to find out the number of turns. Once the diameter $(d)$ and number of turns $(n)$ are calculated, the remaining design parameters are derived.

3.1. Comparison of Linear and Nonlinear Approaches. Using (1) and (8), a comparison can be made between linear and nonlinear analyses based on design parameters using sample calculations. From (1), by providing the values of required maximum actuation force $\left(P_{\max }\right)$, spring index $(C)$, and transformation stress $\left(\tau_{c}\right)$, we can calculate the required wire diameter. Similarly, from (8), we can calculate the required wire diameter for the nonlinear zone by providing the above

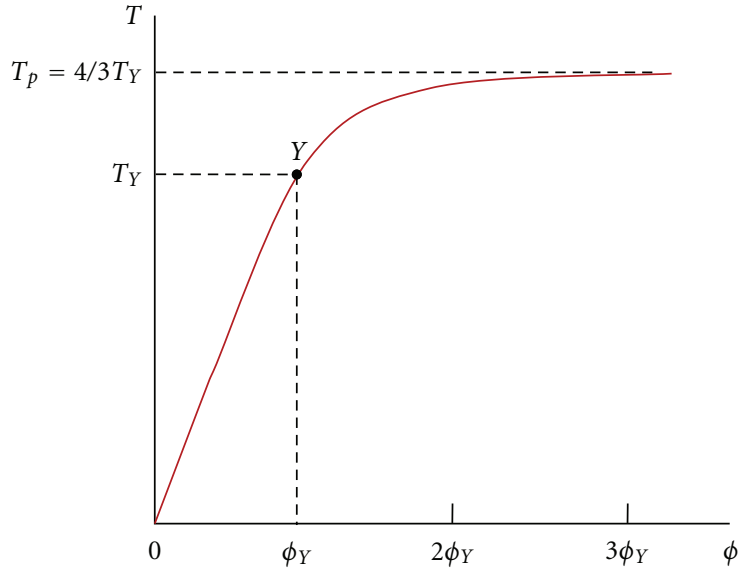

FIgURE 7: Torque versus angle of twist.

parameters along with the total strain $(\gamma)$ value. In Figure 8, the variation of wire diameter $(d)$ with respect to strain $(\gamma)$ at a constant deformation ( $\delta 5=5 \mathrm{~mm}$ deformation) and for different actuation force values $(P=5 \mathrm{~N}, 10 \mathrm{~N}, 15 \mathrm{~N}, 20 \mathrm{~N})$ is shown. The transformation shear stress was considered to be $250 \mathrm{MPa}$ and the strain at the end of linear zone was considered as $2 \%$. The spring index was assumed to be 6. Using (2), the number of turns ( $n$ ) can be calculated by providing the values of wire diameter $(d)$, deformation $(\delta)$ and the total strain $(\gamma)$. Using the same equation, relationship between strain and the number of turns $(n)$ can be obtained which is plotted for different actuation force values. Number of turns is directly proportional to the deformation $(\delta)$ with $\delta=5 \mathrm{~mm}$.

From Figure 8, it is noted that as the strain enters into transformation region $(>2 \%)$, there is an abrupt drop in the design diameter till strain reaches a value of around $3 \%$. Then, it stabilizes to a particular value for higher strains. 


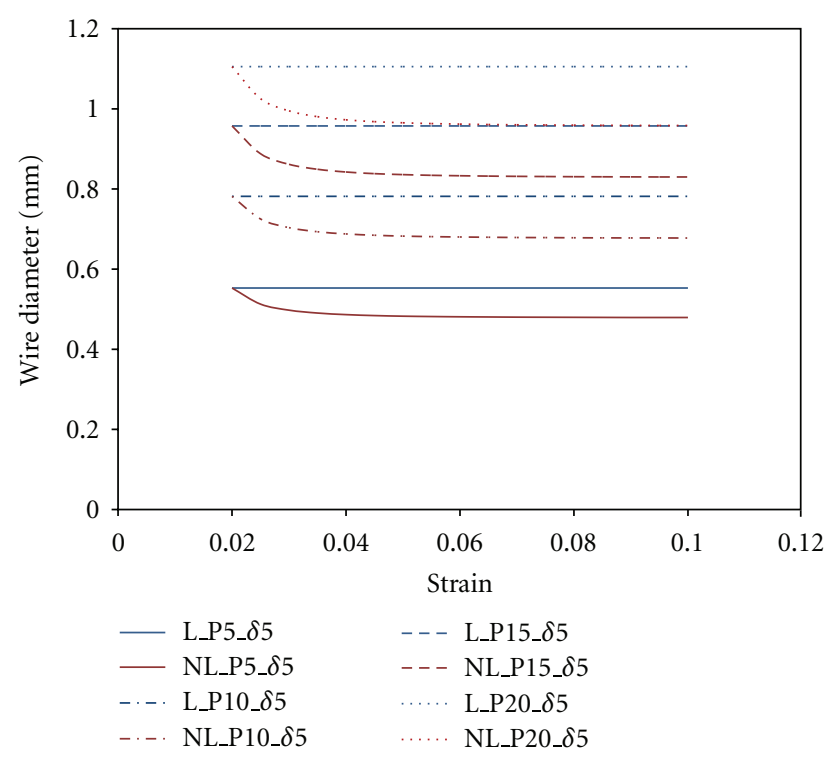

FIgURE 8: Variation of diameter $(d)$ with respect to total strain in martensite phase.

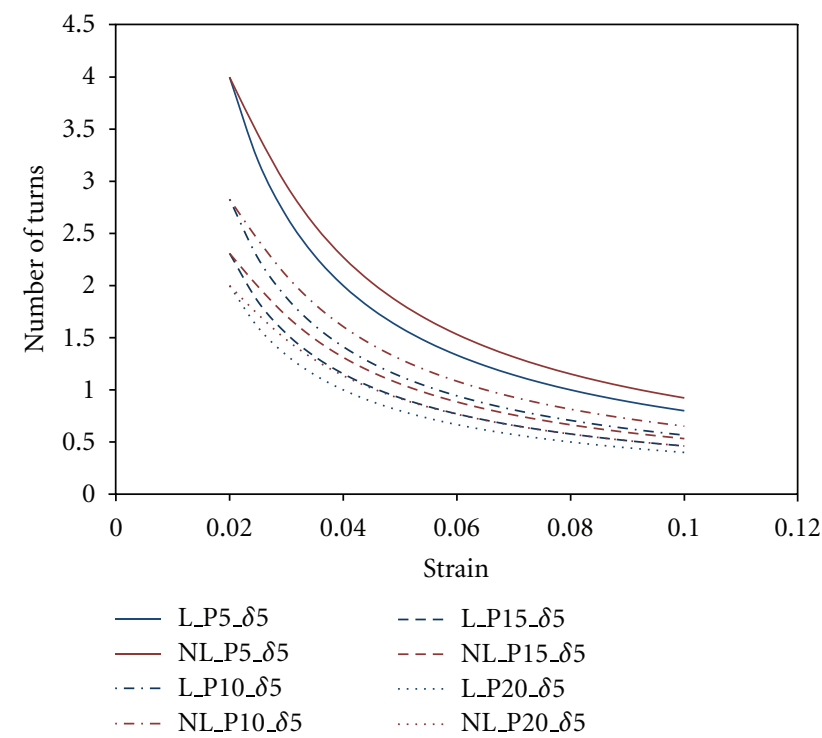

FIGURE 9: Variation of " $n$ " with respect to total strain in martensite phase.

It can be noted that as for higher strain values, (8) asymptotically reduces to

$$
P=\frac{2}{3} \frac{\pi}{C} c^{2} \tau_{y}
$$

So, for large values of shear strain, the wire radius can be found out by simplifying the above equation as

$$
c=\sqrt{\frac{3 F}{2 \pi \tau_{y}}} .
$$

Figure 9 shows that the required number of turns variation follows the same trend as required wire diameter as the observed in Figure 8. While the difference between the two models is considerable for lower values of strain, it comes down and stabilizes for higher strain values. However, values obtained from the nonlinear model are more than the linear model values due to the fact that number of turns $(n)$ is inversely proportional to the wire diameter $(d)-(2)$. Also, with increase in the actuation force, the difference between two curves is coming down. Thus, when transformation strain is taken into consideration, it is suggested to use (11) and (2) for the calculating design parameters.

\section{Conclusions}

The design parameters are analyzed in the paper with the consideration of transformation strains in the design of SMA actuator springs. An idealized nonlinear behavior of SMA is used to reduce the complexity in the formulation and is compared with the traditional linear approach. Additionally, external hard stops are assumed in the analysis to constrain for allowable total transformation strain that is closely associated with the fatigue life of the spring. While the required wire diameter for the same actuation force is lower when nonlinear behavior is considered, the number of turns needs is higher for the same stroke. It is shown that the effect of transformation strain can be taken into account in the design procedure modified for the nonlinear behavior of the SMA spring. New simplified relations are also obtained for higher strain values. Further studies have to be carried out to quantitatively define the fatigue-sensitive problems associated with the transformation strain.

\section{References}

[1] D. J. Leo, Engineering Analysis of Smart Material Systems, John Wiley \& Sons, New York, NY, USA, 2007.

[2] R. A. A. de Aguiar et al., "Shape memory alloy helical springs: modeling, simulation and experimental analysis," in $A B C M$ Symposium Series in Solid Mechanics in Brazil, vol. 2, pp. 169$182,2009$.

[3] H. P. Han, K. K. Ang, Q. Wang, and F. Taheri, "Buckling enhancement of epoxy columns using embedded shape memory alloy spring actuators," Composite Structures, vol. 72, no. 2, pp. 200-211, 2006.

[4] H. J. Lee and J. J. Lee, "Evaluation of the characteristics of a shape memory alloy spring actuator," Smart Materials and Structures, vol. 9, no. 6, pp. 817-823, 2000.

[5] I. Spinella and E. Dragoni, "Analysis and design of hollow helical springs for shape memory actuators," Journal of Intelligent Material Systems and Structures, vol. 21, no. 2, pp. 185-199, 2010.

[6] T. C. Waram, Actuator Design Using Shape Memory Alloys, Tom Waram, Ontario, Canada, 2nd edition, 1993. 

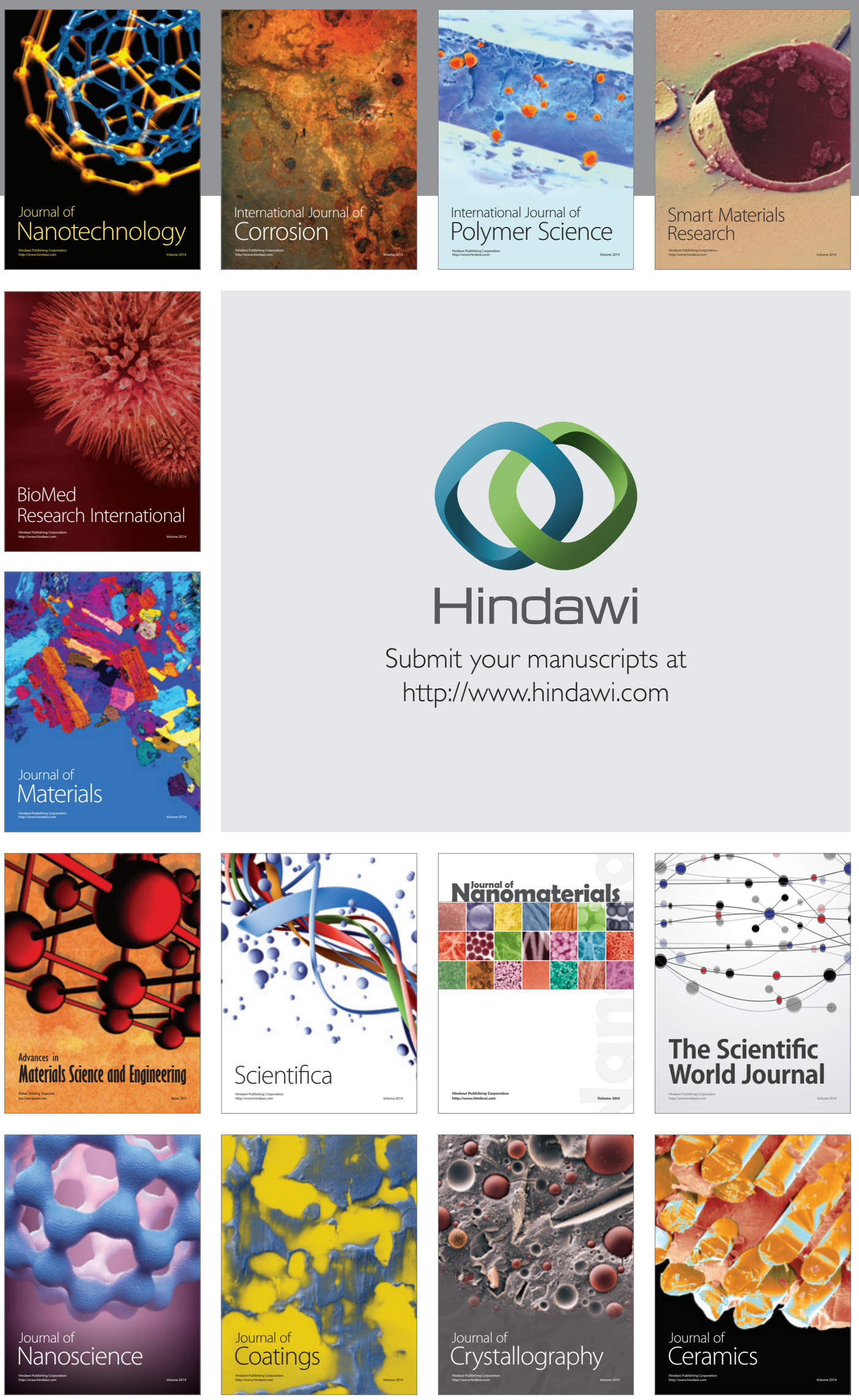

The Scientific World Journal

Submit your manuscripts at

http://www.hindawi.com

\section{World Journal}

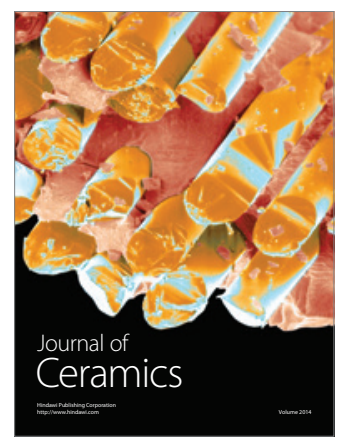

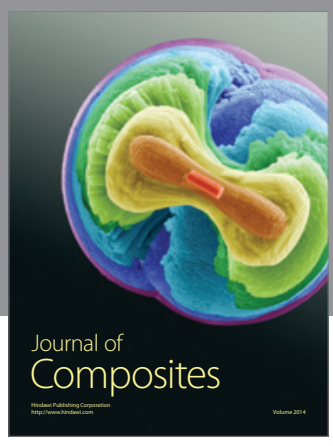
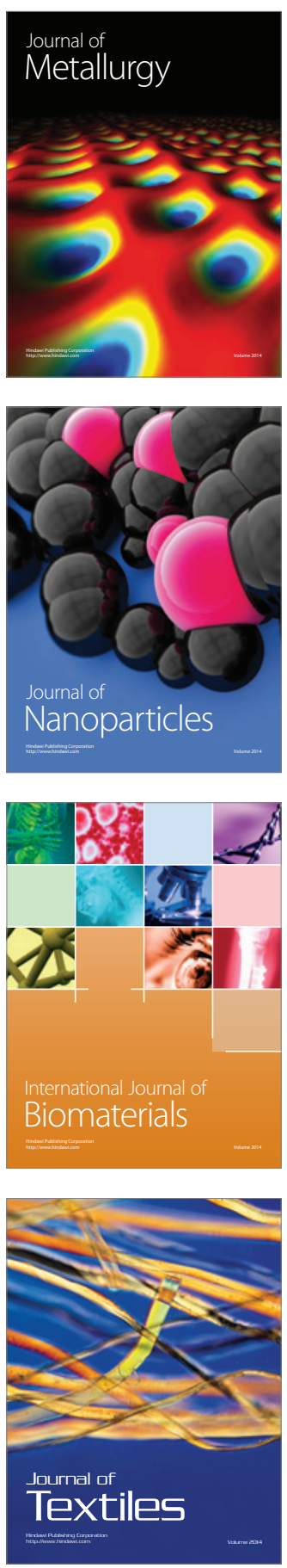